\title{
Apuntes sobre Periodismo de Investigación en el Cono Sur ${ }^{1}$
}

\section{Some notes on Investigative journalism in Southern America}

\author{
Claudia Lagos \\ Universidad de Chile \\ cllagos@uchile.cl
}

\begin{abstract}
Resumen
El presente artículo analiza las principales tendencias temáticas y metodológicas, así como los problemas más relevantes que enfrenta el periodismo de investigación en el Cono Sur (a excepción de Brasil). A partir del análisis descriptivo de reportajes de investigación realizados durante la última década en la subregión y sus respectivos resúmenes metodológicos; así como de entrevistas semiestructuradas con periodistas y especialistas en el área, se ofrecen algunos apuntes de lo que ha sido este género periodístico en la última década y las perspectivas que se abren.
\end{abstract}

Palabras Clave: Periodismo de investigación, Cono Sur, democracia.

\begin{abstract}
This article analyzes the main trends, themes and methods of investigative journalism in the Southern Cone (except for Brazil). More than twenty stories and their methodological summaries were analized, as well as semi-structured interviews with journalists and experts in the field. This article propose some notes on what has been this kind of journalism in the last decade and the prospects that open.
\end{abstract}

Keywords: Investigative journalism, South America, democracy.

\footnotetext{
1 Una primera versión de este trabajo fue presentada en la Conferencia Latinoamericana de Periodismo de Investigación COLPIN, celebrada en Bogotá en octubre de 2012, por encargo del Instituto Prensa y Sociedad (IPYS), que solicitó a la autora revisar, sistematizar y analizar los reportajes de periodismo de investigación de los países incluidos en el artículo y que participaron en el Premio Latinoamericano a la Mejor Investigación Periodística que entregan anualmente IPYS, Transparencia Internacional para América latina y El Caribe y Open Society Institutes.
} 


\section{INTRODUCCIÓN}

"Ningún tema está agotado; nada está jamás totalmente dicho"

Periodista argentina

El periodismo de investigación en América Latina ha permitido encarcelar a presidentes o ex presidentes y políticos. En los últimos 15 años, se han conocido escándalos de corrupción y violaciones a los derechos humanos en México, Costa Rica, Guatemala, Perú, Chile, Argentina y Brasil (Cárdenas y González, 2006). La prensa ha jugado un rol clave en develar comportamientos ilegales y antiéticos de las principales autoridades de los países de la región. En cada caso, los reportajes han impactado en dos sentidos: primero, se convirtieron en especies de Watergates latinoamericanos, desafiando a las instituciones, persiguiendo a figuras poderosas y exigiendo reformas. Y, en segundo lugar, han transformado el modo en el cual entendemos el periodismo de investigación latinoamericano y han influido en él hasta nuestros días. Además, la proyección y consecuencias de estas historias se han multiplicado debido a la mediatización de nuestras sociedades.

El impacto de estos casos se entiende no solo por las características que en sí mismos tienen y los actores que han involucrado, sino que también porque en muchas ocasiones, la prensa y los medios han ejercido - a propósito o no- la función que debían asumir instituciones como el Poder Judicial, por ejemplo. Allí donde supuestamente las instituciones debieran funcionar, muchas veces el periodismo ha ocupado su lugar.

Todos los casos mencionados recuerdan el caso Watergate y la serie de notas y reportajes publicada por The Washington Post en los '702 que contribuyeron a la renuncia de Richard Nixon a la presidencia de Estados Unidos en 1974: dos jóvenes periodistas del periódico -Robert Woodward y Carl Bernstein- descubrieron un plan ilegal y antiético diseñado desde la misma Casa Blanca y que perseguía sabotear al candidato demócrata a la presidencia, George McGovern. Para ello, Woodward y Berstein siguieron metódicamente pistas y enfrentaron enormes obstáculos (Bernstein y Woodward, 1984). Este conocido caso es el canon del periodismo de investigación y es el modelo usado por los reporteros latinoamericanos en los ejemplos más arriba señalados.

Watergate y sus réplicas latinoamericanas pueden intimidar a quien quiera animarse a hacer periodismo de investigación. Sin embargo, este género periodístico no solo ha contribuido a que presidentes renuncien; hay ejemplos que han apuntado a otros objetivos igual de relevantes para las democracias de la región.

2 Debido al nombre del edificio en Washington D.C. 
Como dice John Dinges, “[p]arece increíble, pero en Estados Unidos las mejores investigaciones se hacen en televisoras locales $[. .$.$] . El caso de las camionetas Ford$ Explorer, por ejemplo, que lo destapó una televisora en Texas. O una estación en Utah que descubrió que un alcalde viajaba a Nueva York para reuniones oficiales y se quedaba cinco días yendo al teatro, divirtiéndose" (entrevistado por Scharfenberg, en Cañizales, 2006, p. 61).

El origen del periodismo de investigación lo encontramos en la época de los muckrackers (o husmeadores de basura), hacia fines del siglo XIX en Estados Unidos (García Luna, citado en Martínez, 2004, p. 25):

La diferencia básica que existe entre los muckrackersy los periodistas de investigación contemporáneos radica en que los primeros practicaban un periodismo de reivindicación que los impulsaba a tomar posturas políticas sumamente marcadas. Si bien en la actualidad el periodismo de investigación sigue basándose primordialmente en remover basura -aunque esta sea ahora basura electrónica-, no todos los periodistas de investigación son muckrackers. En rigor, sus trabajos mezclan la exposición descriptiva de los hechos con la demanda de reforma social y, en algunos casos, con la toma de cierta posición política” (García Luna, citado en Martínez, 2004, p. 26).

El periodismo de investigación tal y como lo conocemos hoy nace en la década del '70, donde el caso Watergate es el hito principal. Como explica John Dinges, "[e]l periodismo de investigación realmente nació como género en los años '70 y se desparramó por todo el mundo como una meta periodística que no existía antes" (entrevistado por Scharfenberg, en Cañizales, 2006, p. 60).

Los principios ordenadores de este género son: "cómo obtener los datos (fuentes), cómo procesar la información recogida (técnica) y cómo presentar los resultados (informes)" (Martínez, 2004, p. 21).

El presente trabajo describe y analiza el periodismo de investigación en el Cono Sur (menos Brasil), a partir de los trabajos ganadores y finalistas durante una década del Premio a la Mejor Investigación Periodística que entregan anualmente el Instituto Prensa y Sociedad (IPYS) y Transparencia Internacional para América Latina y El Caribe, con el apoyo de Open Society Institutes.

Este artículo está dividido en tres secciones: la primera, revisa las principales tendencias temáticas, metodológicas y de producción que caracterizan los artículos y trabajos de investigación incluidos en esta revisión; la segunda, analiza las percepciones de informantes clave (periodistas, editores y académicos de cuatro países incluidos en el análisis) y, finalmente, se ofrecen algunas reflexiones finales. 


\section{TEMAS, MÉTODOS Y PRODUCCIÓN}

Hasta el año 2012, una década después de su primera versión, el Premio anual a la Mejor Investigación Periodística en América Latina nunca había sido evaluado. Sus organizadores -el Instituto Prensa y Sociedad (IPYS), Transparencia Internacional para América Latina y El Caribe y Open Society Institutes- convocaron a un panel de expertos en estudios de periodismo con el objetivo de indagar si el premio estaba cumpliendo su objetivo de incentivar este tipo de periodismo en el continente.

Otro objetivo de esta evaluación era averiguar cómo mejorar el premio como una herramienta para fortalecer el periodismo de investigación en América Latina. Para responder a ambos propósitos, IPYS entregó a los consultores externos los artículos originales premiados así como los finalistas, los formularios descriptivosmetodológicos de cada trabajo y las transcripciones de entrevistas semiestructuradas con informantes clave. A cada experto se le asignaron todos los trabajos de un país o de un conjunto de países y trabajó de manera independiente. El equipo contó con un coordinador.

Se analizaron más de cien reportajes originales, los que provenían del archivo de finalistas y ganadores del premio desde su primera versión, en 2002. Resumidamente, de los 31 reportajes o series de artículos premiados desde la primera versión del premio en 2002-2003, siete de ellos son brasileros y solo cinco corresponden a países del Cono Sur distintos a Brasil, y que fueron publicados en medios de Paraguay y Argentina. Ningún trabajo chileno o uruguayo ha obtenido alguno de los tres primeros lugares desde que se entrega este premio.

Cada trabajo contaba, además, con un formulario descriptivo metodológico que el autor o los autores debían completar para postular al premio. Este formulario tiene ocho secciones: 1) orígenes de la investigación; 2) hipótesis de la investigación; 3 ) principales hallazgos; 4) metodología; 5) fuentes utilizadas; 6) recursos (personal involucrado, recursos financieros, tiempo); 7) obstáculos (amenazas o procesos judiciales); y 8) el impacto del trabajo (si es que las revelaciones del trabajo motivaron investigaciones oficiales, procesos judiciales, destitución de funcionarios involucrados o reformas legales, por ejemplo).

Además de los trabajos originales y los formularios descriptivos-metodológicos, miembros del equipo de IPYS entrevistaron a informantes clave (periodistas, editores y académicos) con una pauta semiestructurada. Las transcripciones completas también fueron parte del material analizado por los consultores externos.

Los asesores tuvieron completa libertad de elegir el enfoque con el cual analizar los trabajos originales, así como el resto del material. Para este artículo en particular, se usó un enfoque de análisis temático tanto para los trabajos originales, como para los formularios descriptivos-metodológicos y las entrevistas a informantes clave. Fue un proceso inductivo. 
Como parte del panel de consultores, se analizaron 24 reportajes originales y sus respectivos formularios de Argentina, Chile y Paraguay, así como 31 entrevistas transcritas de informantes clave de esos mismos países, más Uruguay.

Este artículo tuvo dos propósitos: primero, señalar las principales características del periodismo de investigación que se hacía en la pasada década en el Cono Sur (a excepción de Brasil), considerando factores como el soporte, género, autores, temas, el tiempo invertido en la investigación, basados en los trabajos mismos; segundo, explorar las percepciones de informantes clave sobre el periodismo de investigación en sus países.

\section{ANÁLISIS DE ARTíCULOS}

En esta sección, explicaremos las principales características del periodismo de investigación en el Cono Sur (excluyendo Brasil) en la última década, tomando en consideración tanto los trabajos mismos como sus formularios descriptivos. Esta sección pretende ofrecer una imagen panorámica del periodismo de investigación en estos países así como indagar en ciertas claves para profundizar en el conocimiento de éste.

De acuerdo con estos datos, los trabajos premiados están desbalanceados según países. Argentina ha sido seleccionada catorce veces, ya sea como finalista o ganador. En tres ocasiones, trabajos realizados en medios argentinos han sido galardonados con uno de los tres primeros lugares del premio. En el mismo período analizado, seis trabajos publicados en Paraguay y cuatro, en Chile, fueron finalistas o ganadores. No ha habido ningún artículo uruguayo entre los finalistas o ganadores desde la primera versión del premio hasta ahora.

Tras analizar 24 artículos, el soporte más común para el periodismo de investigación en estos países es el periódico. La mayoría de los reportajes (17) se publicó como una serie de artículos en diarios. Dos investigaciones aparecieron en revistas, cuatro fueron publicadas online, y solo una fue emitida por televisión. Ninguna de las investigaciones apareció en radio. Es importante señalar que de los 24 artículos finalistas o ganadores en Argentina, Paraguay y Chile en diez años del premio considerados para esta revisión, solo uno fue publicado en un medio local.

El análisis también demuestra tendencias de masculinización e individualismo entre los periodistas de investigación de estos países. Hay 33 autores para los 24 artículos analizados, de los cuales solo 9 son mujeres y 20 artículos fueron firmados por un autor.

El concurso nació y se mantuvo por varios años para premiar a la mejor investigación de un caso de corrupción en el continente. Solo en años recientes, la convocatoria se amplió temáticamente y considera distintas problemáticas. Dicho 
origen explica una sobrerrepresentación de casos de corrupción, cohecho, abuso de cargos públicos y malversación de fondos, entre otras malas prácticas, como temas y problemas que ha abordado el periodismo de investigación en el cono sur.

Eso también explica que la mayoría de los trabajos devele malas prácticas en los poderes del Estado y desnude delitos o faltas a la ética de funcionarios públicos (presidentes, ministros, candidatos a cargos públicos, máximas autoridades de empresas estatales, servicios especializados del Estado de carácter intermedio, presidente del Congreso, entre otros), así como de representantes del sector privado (para que se verifique una mala práctica en el sector público, muchas veces es necesaria la connivencia de empresarios o representantes de otras organizaciones intermedias, como sindicatos). Fue el caso de empresas transnacionales y presidentes de sindicatos.

Las investigaciones detrás de los reportajes o series de reportajes acá analizados demoraron al menos dos años. Al menos cinco de los trabajos analizados, se extendieron por más de un año.

Entrelas herramientas utilizadas, severifica una gran variedad: reporteo tradicional, muchas y diversas fuentes, algunas de las cuales fueron clave $y / u$ originaron la investigación; uso de leyes de acceso a la información; uso de herramientas digitales y periodismo asistido por computadora; acceso a bases de datos públicas y privadas; grabaciones con cámaras o grabadoras ocultas. Del mismo modo, en algunos casos los periodistas omitieron su condición u oficio y se presentaron ante las fuentes como afectados. Según lo señalado por los autores de las investigaciones analizadas, ninguno pagó por información, aunque en más de un caso una o más fuentes sugirieron esa posibilidad.

\section{PERCEPCIONES INFORMANTES CLAVE}

Junto con los trabajos originales y las guías descriptivas metodológicas de estos, se tuvieron a la vista 31 entrevistas semiestructuradasa igual númerodeinformantes clave. En la medida que los objetivos del presente trabajo eran evaluar el funcionamiento del premio, se incluyeron entre los entrevistados a editores, reporteros y académicos de países donde no se han registrado trabajos ganadores o finalistas, como es el caso de Uruguay. La muestra no es representativa ni probabilística, sino que busca profundizar en tópicos identificados en los reportajes originales, así como en la escasa literatura en la materia en la subregión considerada en este trabajo.

El cuestionario semiestructurado abordaba, entre otros, los siguientes temas: si es que hay o no diferencias con el periodismo de investigación que se realiza en Estados Unidos y Europa y, de haber, cuáles serían estas; cuáles son los valores que debieran premiarse y fomentarse en el periodismo de investigación en el continente; cuáles 
son los temas menos abordados; cuáles son las capacidades que debiera tener un periodista de investigación y cómo fomentarlas en la formación universitaria.

En este apartado, sintetizaremos las principales percepciones de los informantes clave entrevistados, a partir de cuatro grandes temas: 1) El contexto del periodismo en el Cono Sur (exceptuando Brasil), en comparación a Estados Unidos o países de Europa y su evolución/retroceso; 2) los principales valores que debieran fomentarse en el periodismo de investigación en la región; 3) cuáles debieran ser las cualidades que debieran caracterizar al reportero investigativo y 4) cuál sería la evaluación crítica del premio.

\section{Una carrera de obstáculos}

"No es lo mismo premiar a quien puede investigar sentado en una Ferrari, que en un Fiat 1.

A quien utiliza un equipo, como a quien lo hace todo él.

A quien tiene una Nikon full time como a quien tiene una simplecita Kodak".

Periodista uruguayo

El contexto sí importa. El periodismo moderno en Estados Unidos, en general, y el periodismo de investigación, en particular, surge de la mano de una prensa comercial, de gran tirada, que apunta a la clase media ilustrada y con poder adquisitivo de fines del siglo XIX y principios del siglo XX. Sin embargo, en los países de América Latina, el proceso fue mucho más artesanal y es deudor de la prensa de opinión europea, sin garantizar niveles mínimos de independencia de los poderes formales o de facto (Waisbord, 2000).

En América Latina, la prensa surge de la mano de movimientos o partidos políticos o bien cercana al Estado, en tanto este le otorgó generosos subsidios para surgir. Del mismo modo, la participación de los Estados en los sistemas de medios, sobre todo en el caso de las concesiones radioeléctricas, también se caracteriza por una intervención importante en sus industrias infocomunicacionales (Mastrini y Becerra, 2006).

Del mismo modo, el sistema político es un factor a considerar en el estadio de desarrollo del periodismo de investigación en América Latina, en general, y en el Cono Sur, en particular.

Mientras el periodismo en Estados Unidos y Europa se caracteriza por haber surgido y haberse desarrollado de la mano del sistema democrático (con las diferencias que cada país tiene y las inflexiones registradas en países como España, Portugal o Grecia, que padecieron dictaduras durante varias décadas en el siglo XX) (Hallin y Papathanassopoulos, 2002), América Latina se caracteriza más bien por sus Estados en proceso de construcción. En algunos casos, Estados fallidos. 
Durante el siglo XX, la enorme mayoría de los países de la región sufrieron dictaduras de distinto signo. En el caso de los países acá analizados, todos vivieron dictaduras militares durante la segunda mitad del siglo XX, con violaciones sistemáticas a los derechos humanos, entre las que se cuentan las restricciones a la libertad de expresión, la persecución de periodistas y comunicadores, el cierre de medios de comunicación o su acorralamiento a través de mecanismos indirectos, entre otros factores. Estas características determinan el tipo de Estado, de organización política y, por lo tanto, de prensa y periodismo que hoy encontramos en cada uno de estos países, con sus especificidades locales.

"Es imposible desarrollar buen periodismo de investigación en sociedades con una baja intensidad democrática o decreciente interés por lo público. El progreso cívico de los países también 'empuja’la calidad de sus periodistas, y produce mejores resultados”. Periodista argentino

Un desarrollo democrático de más larga data y, por lo tanto, más robusto en comparación a los países del Cono Sur, ha permitido también una cultura documental más fuerte que, a su vez, ha sido una herramienta fundamental para el periodismo de investigación, tanto en Estados Unidos como en Europa. Las leyes de acceso a la información en países escandinavos y en Estados Unidos son de larga data, en comparación con las del Cono Sur.

"La enorme disponibilidad de datos y documentos públicos con que cuentan los periodistas estadounidenses, debido a la fuerte cultura documental que tiene la administración pública (es una ventaja)”.

Periodista argentino

Del mismo modo, tanto la producción como el consumo cultural están altamente concentrados y centralizados en las capitales nacionales (Mastrini y Becerra, 2006 y 2009). De hecho, solo uno de los trabajos acá incluidos fue realizado y publicado en un medio regional.

En ese contexto, el periodismo de investigación en los países considerados, si bien se inspira en el modelo estadounidense, tiene sus características -o determinantespropias del contexto político, económico y social en que se desarrolla. Entre los cuatro países analizados, también hay diferencias, según se desprende de las percepciones de los informantes clave. Del mismo modo, el periodismo de investigación latinoamericano se diferencia del norteamericano europeo -según los entrevistadosdebido al acceso a menos recursos económicos, de apoyo y capacitación. Para algunos, los profesionales del continente suplen la precariedad con ingenio. 
"Algunas de las diferencias fundamentales ... siguen teniendo que ver con el contexto en el se desempeña la prensa. Si bien en algunos países latinoamericanos ya existen leyes de acceso a la información pública, su implementación es lenta en algunos casos y, en otros, ineficaz. En otros países las leyes de acceso a la información son una cuenta pendiente y es allí donde las investigaciones muchas veces dependen de una filtración.... El contexto legal también resulta determinante.... En muchos de los países del hemisferio aún existen leyes de difamación penal ... En países donde la violencia de grupos del crimen organizado es generalizada, y en ocasiones actúan en colusión con la autoridad, los periodistas que realizan investigación enfrentan graves riesgos y en muchos casos ponen en peligros sus vidas”.

Periodista argentino

"No le temo a ningún periodista con 29 años de carrera como yo, que trabaje en Europa o en Estados Unidos. Trascienden más, porque son periodistas de grandes medios. Seguro nunca sufrirán un atentado o una denuncia penal".

Periodista uruguayo

"La redacción de salas de periodismo más avanzadas es clara, contundente, con notas de colores que impactan, que pegan, que conmueven. En América Latina hay quizá descubrimientos más valiosos, gente con más coraje, pero con poca luz a la hora de presentar esos materiales ante la opinión pública”.

Periodista paraguaya

Otro factor relevante para los entrevistados es el nivel de polarización política en los países considerados en este análisis, contexto del cual no escapan los medios de comunicación y, en particular, el periodismo. Esta percepción se verifica principalmente entre los informantes clave argentinos consultados.

"La politización que existe en América Latina no hace más que hacer más permeable a la labor periodística de ataques políticos así como de entes de control como es el caso de la publicidad oficial encubierta".

Periodista argentina

"Hay poco periodismo de investigación, creo que principalmente por un cuestión de presupuesto y costos. Los medios no cuentan con plantillas muy grandes de periodistas, por lo que estos deben dedicarse a escribir artículos a diario y destinan poco tiempo a investigaciones de fondo. No hay departamentos en los medios dedicados a la investigación".

Periodista uruguaya

Así, los informantes clave consultados para este trabajo concuerdan, en su mayoría, en que el periodismo de investigación en el Cono Sur (exceptuando Brasil) ha disminuido o se ha estancado. Y esto es posible explicarlo considerando 
al menos tres factores: los sociopolíticos o económicos (extraorganizacionales), que responden a las condiciones sociales, políticas, económicas de una sociedad, en un momento determinado, en que se desenvuelve el periodismo de investigación; factores organizacionales, es decir, aquellos que dependen de las decisiones al interior de los medios de comunicación; y los factores profesionales o individuales, siguiendo el modelo de niveles de influencia propuesto por Shoemaker y Reese (1996).

Entre los factores extraorganizacionales, se distingue la polarización política, como advierte buena parte de los entrevistados argentinos; la concentración de la propiedad de los medios de comunicación, que incluye la distribución de la pauta publicitaria y las presiones económicas y judiciales derivadas de ésta; la oferta gratuita de medios online, que compite con los medios pagados.

"La calidad de muchos trabajos decayó a causa del cerrojo informativo impuesto por el gobierno argentino desde hace cuatro años: muchas estadísticas oficiales son falsas, todos los funcionarios tienen la orden de no atender ni responder los requerimientos periodísticos de algunos medios (principalmente los diarios Clarín y La Nación), los periodistas no tenemos acceso a ningún documento público y los pedidos de acceso a la información (débilmente amparados en un decreto de 2003, cuando aún no existe una ley nacional que contemple ese derecho humano) ya no son respondidos. Estas restricciones afectan la calidad del trabajo".

Periodista argentino

"En Uruguay la corrupción política ha dejado de ser investigada desde que hubo un cambio de fuerza política. Se investiga algo, pero no se llega hasta el fondo en casi ningún tema. Algo similar ocurre con los grandes negocios de inversiones extranjeras que se instalan en el país”.

Periodista uruguayo

Entre los factores organizacionales que los entrevistados perciben como relevantes para explicar el deterioro y/o estancamiento del periodismo de investigación en estos países, se cuenta la decisión de los medios de disolver equipos de investigación periodística, disminuir su financiamiento y no costear gastos derivados de investigaciones (como el acceso a bases de datos o pago de informes comerciales, por ejemplo).

"La gran debilidad está asociada al mundo televisivo, históricamente poseedor de espacios dedicados al periodismo de investigación. La búsqueda por captar a la mayor cantidad de audiencia y rebajar los costos de producción, está llevando a los ejecutivos de los canales chilenos a desistir de este género periodístico. En otros casos se reduce a tal punto el presupuesto de estos equipos periodísticos, que se hace imposible llevar adelante investigaciones de gran calidad e impacto".

Periodista chileno 
"Entre los motivos figuran cuestiones muy prácticas, como la designación de principales investigadores en otros cargos: encargados regionales, responsables del diario digital, secretarios de redacción, presentadores de noticias en TV y otros, además de la casi desaparición de los equipos de investigación en televisión”.

Periodista paraguayo

"Los medios no invierten en reporteros de investigación, tienen pocos, no hay equipos consolidados trabajando para producir metódicamente".

Periodista paraguaya

Entre los factores profesionales o individuales que podrían estar afectando el desarrollo del periodismo de investigación en los países analizados, según los entrevistados se cuentan la falta de capacitación, cierto afán de protagonismo de algunos reporteros; $y$ una agenda restringida o poco creativa.

\section{Temas/perspectivas ausentes}

Cuandolos medios publican algo, dejan un universo de situaciones, actores, problemas fuera del marco, fuera de la agenda. En el caso del periodismo de investigación, Silvio Waisbord (2000) destaca que en Sudamérica ha sido prolífico en abordar cuestiones referidas al poder estatal. Y eso no sería de extrañar, dice, toda vez que las prácticas y rutinas periodísticas recurrentes apuntan a reportear al Estado. Por lo tanto, las fuentes recurrentes, los temas más cercanos para el periodismo del subcontinente, han estado vinculadas al Estado, las malas prácticas de sus funcionarios, etc. Y esto cambia al vaivén de los cambios políticos también.

"En Uruguay hay un franco retroceso en la práctica del periodismo de investigación. La razón más importante es que los medios electrónicos nunca le dieron demasiado espacio a esta tarea y tanto periódicos como revistas y semanarios, en papel, (que sí lo hacían) han optado por la política del 'no te metás' que, para algunos empresarios, parece ser más apetecible y redituable desde el punto de vista económico”.

Periodista uruguayo

Del mismo modo, no hay que olvidar que la sustentabilidad de los medios de comunicación contemporáneos se debe al carácter comercial de estos, viven de la publicidad y de la captación de audiencias. A su vez, los medios forman parte de grandes conglomerados económicos que tienen intereses cruzados en otras áreas de la economía de nuestros países. Estos elementos dificultarían al periodismo de investigación abordar ciertos problemas, como los llamados delitos de cuello blanco. 
"Los intereses de las grandes compañías multinacionales en la región son motivo de escasa investigación. Se pone mucho la lupa en los gobiernos, como debe ser, pero poco en las corporaciones económicas, debido, creo yo, a su relación con la publicidad de los medios. El sector financiero, en este contexto, es muy poco investigado".

Periodista argentino

En este ámbito, los entrevistados distinguen como parte de los grandes intereses económicos aquellos relacionados a las actividades extractivas de la economía (la minería, los derechos de agua, entre otras), las empresas farmacéuticas; el sector bancario, las multinacionales, intereses de los grupos energéticos, la venta de armamentos, y, en particular, la relación entre el sector privado-empresarial y los gobiernos, y en particular, el financiamiento de la política. En este conjunto de preocupaciones se incluye también la falta de fiscalización o de seguimiento al mundo de los poderosos en términos generales, considerando las enormes desigualdades sociales de los países incluidos en esta revisión.

En el otro lado de la balanza, en medios de comunicación que sobreviven en condiciones económicas y empresariales más precarias, es difícil abordar temáticas difíciles de investigar, por sus posibles consecuencias o por los altos costos que involucrarían.

Temáticamente, los entrevistados distinguen una larga lista de problemas que afectan a los países de la región y que no están siendo objeto de investigación periodística sistemática. Estos son posible organizar en al menos dos grandes grupos: uno, referido a prácticas ilegales o antiéticas, y otro, que abarca cuestiones de derechos de las personas.

Entre los primeros, se cuentan el lavado de dinero, la explotación sexual de niños y adolescentes, el narcotráfico, el tráfico de armas y de personas, el comercio ilegal de la frontera.

En el segundo grupo, los entrevistados llaman la atención sobre la poca cobertura o interés del periodismo de investigación en abordar cuestiones referidas a derechos de las personas, como la salud, la educación y el medioambiente, entre otros.

Finalmente, un elemento que aparece en relación a los valores que debieran fomentarse en el periodismo de investigación y que es importante destacar:

"El periodismo está cada vez más lejos de la gente común, de la calle. Más aún desde el boom de Internet".

Periodista argentino

\section{Lo más valioso}

A los entrevistados se les preguntó si estaban de acuerdo con los criterios que, a la fecha, se han premiado -esfuerzo de búsqueda, impacto y presentación-; cuáles 
debieran ser los valores a destacar y si hay algunos que debieran considerarse y que, hasta ahora, no se han incluido.

Considerando estas tres dimensiones, agrupamos los elementos que los entrevistados consideran que debieran valorarse en el periodismo de investigación en estos países en tres dimensiones: valores transversales (como integridad o respeto a las personas); valores profesionales (como el profesionalismo o la persistencia) y cuestiones técnicas (como el númeroy calidad de fuentes o la metodología empleada).

En el primer conjunto de valores -transversales-, que los entrevistados consideran son los más importantes, se cuentan la integridad, el respeto por las personas involucradas; la ética, y el coraje.

En el caso de los valores profesionales, los entrevistados concuerdan en que estos deben considerar el profesionalismo; la variedad, importancia y calidad de las fuentes; el esfuerzo, la persistencia, la calidad de la presentación (narración, prosa, guión y estilo); la trayectoria; el perfeccionamiento; el rigor; la iniciativa; los métodos de trabajo; la independencia.

Todo ello, en un contexto que también debiera ser considerado a la hora de valorar las investigaciones periodísticas. A saber: relevancia del tema, aporte social de la investigación; los obstáculos superados; la repercusión del trabajo en la sociedad o en una parte de esta; importancia de los personajes investigados.

"La dificultad (obstáculos del poder) y los riesgos (incluso de vida) que implica conseguir determinada información, así como la trascendencia y las consecuencias que genera la publicación. El factor sorpresa -de pronto denunciar a un personaje olvidado por los grandes medios-, la perplejidad que genera. En América Latina, cualquier investigación que desnude las grandes deudas de la "democracia" y las fallas estructurales debe ser atendida. Lo novedoso en la forma de contarlo es un punto extra”.

Periodista argentino

"Lo más importante es el esfuerzo de búsqueda... Me parece relevante observar el contexto vinculado con posibles obstáculos adicionales a la investigación en sí: por ejemplo, el medio para el que el periodista trabaja o si es free lance, la actitud oscurantista de gente poderosa alrededor de lo investigado; las formas y estrategias para acceder a fuentes renuentes. Muchas veces estos puntos son claves para entender resultados de la investigación".

Periodista argentino

"El contexto del país en que se investiga: no es lo mismo Uruguay con Mujica y Venezuela con Chávez".

Periodista argentino 
"Una investigación puede ser muy simple o muy compleja, pero su importancia está dada por las connotaciones políticas, económicas y sociales que tuvo esa investigación”.

Periodista paraguayo

"No se le debe dar demasiada importancia al impacto de la información y sí se debe priorizar la calidad de la información y la forma en que esta es relatada".

Periodista uruguaya

"Se debiera premiar el coraje de aquellos colegas que, sin contar con el respaldo de grandes medios, tratan de hacer periodismo de investigación”.

Periodista chileno

\section{Lo que debe tener o cultivar un periodista de investigación}

El periodismo en el último decenio ha cambiado vertiginosamente: se han desarrollado las nuevas tecnologías, han cambiado las audiencias, ha disminuido el interés por leer y consumir periódicos y, en los países acá analizados, la televisión sigue siendo el soporte más relevante y de mayor impacto y consumo.

Se ha ampliado la oferta de formación universitaria en comunicación y periodismo, al mismo tiempo que se ha precarizado su ejercicio profesional.

En ese contexto, ¿cuáles son las características que debe tener y cultivar un periodista de investigación? ¿Cuáles son las habilidades que deben potenciarse en la formación profesional de los futuros periodistas, pensando en que se desempeñen en el ámbito de la investigación?

$\mathrm{Al}$ igual que en el caso de los valores o criterios que debieran promoverse en el periodismo de investigación, las capacidades que debiera tener un periodista en este ámbito, así como las que debieran fomentarse en la formación de los futuros reporteros, también pueden agruparse en tres grandes dimensiones: 1) las capacidades transversales o humanistas, 2) las capacidades técnicas, y 3) las capacidades profesionales.

En el primer grupo de capacidades que debiera cultivar un periodista de investigación vigente o en formación se cuentan: la empatía y el trato humano; la ética; su formación intelectual, su compromiso social, su moral; la prudencia y la búsqueda de una mejora social; compromiso con la sociedad; paciencia; ecuanimidad; honestidad intelectual; responsabilidad; perspicacia; templanza.

"Estimular el hambre por querer provocar cambios en la sociedad".

Periodista chileno 
"El 'para qué de su investigación .... Esto de entregar su labor a la comunidad, una labor que no debe morir en un libro o en una nota sino que debe seguir haciendo camino. De lo contrario, seguimos alimentando egos".

Periodista argentina

En cuanto a las capacidades técnicas, es evidente la avidez por fomentar el conocimiento y uso de nuevas tecnologías al periodismo de investigación. A ello se suma también la necesidad de desarrollar el buen uso del lenguaje, en cualquier soporte, porque se entiende como fundamental para que un público lo más amplio posible acceda a las historias. Sobre todo considerando que estas, en este ámbito, suelen ser complejas, enrevesadas o, bien, muy técnicas.

"Variantes de metodologías de trabajo y relación con las fuentes, especialmente con fuentes sensibles, como testigos de identidad reservada, amenazados, etc. Además, nuevas herramientas de búsqueda de información documental y conocer las trampas del poder político y económico que un periodista debe sortear".

Periodista argentina

Finalmente, en el ámbito de las capacidades profesionales, se cuentan ser capaces de distinguir y descubrir temas, de ampliar la agenda, de abordarlos de manera novedosa; de ser rigurosos.

\section{Mejorar, ampliar, focalizar}

Uno de los objetivos del presente trabajo es contribuir a afinar los criterios e incorporar otros que, a la fecha, no han sido considerados pero que debieran incluirse, a la luz de los trabajos que han llegado a ser finalistas o premiados y de acuerdo a las percepciones de informantes clave de los países analizados. Nos parece relevante incluir esta dimensión en el análisis porque los premios, y este en particular, buscan promover cierto tipo de periodismo de investigación y puede resultar estimulante para el ejercicio en la subregión. Además, permite conocer o relativizar criterios que no solo este premio, sino que en general, se buscan en el periodismo de investigación. Entre los criterios que debieran ser revisados, el que aparece más recurrentemente es el de impacto.

"El énfasis observado a veces en la necesidad de resultados concretos, entendiendo éstos como caídas de funcionarios o condenas penales. En ciertos países, si algo caracteriza al poder es que nada lo conmueve ni afecta. Entonces, suponer que revelaciones periodísticas claras, contundentes, precisas e inobjetables provoquen 
una renuncia (como sí ocurrió con ocho ministros en Brasil, por ejemplo) resulta casi injusto en ciertos países".

Periodista argentino

Del mismo modo, surgen criterios ausentes hasta ahora y que los entrevistados consideran importante incorporar al destacar la investigación periodística en los países analizados.

Uno es ampliar y distinguir según soportes. En el primer caso, implica considerar las investigaciones periodísticas que han sido publicadas en formato libro.

"Suelen premiar trabajos publicados en medios, pero no en libros, y considero que eso debería de tomarse en cuenta porque hay trabajos que algunos periodistas terminamos publicando en formato libro porque no encontramos espacio en los medios tradicionales".

Periodista argentina

Y, lo segundo, premiar según soporte considerando que es difícil -y, en ocasiones, injusto-compararlos.

"La realización audiovisual requiere una inversión y un esfuerzo en el tiempo muy distinto al que se aplica a una realización escrita. Por ejemplo: un trabajo audiovisual que cumple a cabalidad con los tres criterios de evaluación, debe tener una valoración distinta que aquella investigación escrita que se basa en una secuencia de reportajes y notas publicadas en varios números de revistas o ediciones de periódicos. En otras palabras: el realizador audiovisual tiene una sola bala (reportaje) para dar en el blanco. En cambio, la prensa escrita tiene toda una caja de municiones para alcanzar el mismo objetivo (una secuencia de publicaciones que sumadas hacen una gran investigación)”.

Periodista chileno

Finalmente, se proponen al menos otros cuatro criterios para premiar a futuro: la trayectoria, el esfuerzo en la búsqueda, la presentación y distinguir según medios regionales o nacionales. Esto aparece como relevante, considerando que del total de trabajos analizados, solo uno corresponde a un reportaje publicado en un medio regional.

\section{REFLEXIONES FINALES}

El presente trabajo enfrenta algunas limitaciones a considerar. Estas se deben, principalmente, al origen y producción de los datos analizados (investigaciones periodísticas finalistas y ganadoras de un premio continental): la sobrerrepresentación 
de ciertos temas (corrupción) así como la sobrerrepresentación del periodismo de investigación en Argentina (tanto en los trabajos analizados y sus respectivos formularios descriptivos-metodológicos, como en los entrevistados).

Sin embargo, las limitaciones del trabajo no invalidan los resultados, debido, al menos, a dos cuestiones importantes: la escasa literatura sobre el tema en los países analizados y por tratarse de la primera evaluación del funcionamiento de un premio prestigioso entre los periodistas del continente. En este último punto, a partir principalmente de las percepciones de los informantes clave, es posible identificar características del periodismo de investigación propias de los trabajos de los países de la región que debieran valorarse y que, hasta ahora, no se han considerado. Estas propuestas exploratorias enriquecen los factores que tradicionalmente se han premiado y destacado (como el impacto), y se relacionan con sus contextos.

Al sintetizar el análisis de los trabajos y sus respectivos formularios descriptivosmetodológicos considerados en este artículo, encontramos que la mayoría de los reportajes de investigación en el Cono Sur (a excepción de Brasil) ha sido publicado en diarios, escrito por hombres y abordado la corrupción del poder político, principalmente en los más altos cargos de la administración del Estado, como presidentes y ministros.

Hay una abrumadora subrepresentación regional en la muestra analizada, considerando que solo uno de los 24 reportajes incluidos en este análisis fue publicado en un medio regional. Todo el resto, es producto del trabajo de medios capitalinos.

La baja o nula presencia de trabajos provenientes de medios de países como Chile o Uruguay (este último no registra ni finalistas ni ganadores), así como también la inexistencia de trabajos en formato radial, puede explorar dos explicaciones: la primera, es que el nivel de investigación periodística en Chile y Uruguay, así como en formato radial, es precaria. $\mathrm{O}$, bien, también puede indagarse en que el premio no ha conseguido difundirse de manera masiva, en toda la región ni en medios de distintos formatos. El presente trabajo carece de los elementos necesarios para responder a este vacío y otros trabajos debieran explorar en este.

Los informantes clave entrevistados perciben al menos dos tenencias que, a primera vista, pueden parecer contradictorias: la primera, es que perciben una disminución o un estancamiento en la investigación periodística en cada uno de los países donde se desempeñan, con un especial énfasis en que dicha baja ha afectado a los medios tradicionales. La segunda tendencia que se encuentra entre los entrevistados se refiere a que es posible advertir un impulso en el periodismo de investigación debido al mayor conocimiento de este en otros países del mundo, incluyendo otros países del continente que - poco a poco- ha permitido intercambiar experiencias, métodos, herramientas de investigación. Casos como el de Brasil -que no se incluye este trabajo - o el de la incorporación de herramientas novedosas -como el periodismo asistido por computadora o de datos, que queda fuera del período de estudio- 
debiera ser analizado en otros trabajos y comparado con la trayectoria y desarrollo del periodismo de investigación en los países acá considerados.

Del mismo modo, desconocemos la dimensión e importancia que tienen otros soportes -principalmente libros- en la difusión de investigaciones periodísticas como estrategia alternativa a los grandes medios que, a juicio de los entrevistados, han disminuido sus recursos y apoyos destinados a la investigación periodística.

Finalmente, uno de los elementos puestos en tensión tanto por los trabajos analizados como por las percepciones de los entrevistados se relaciona con los factores que debieran valorarse o relativizarse. Entre estos últimos, el que provoca tal vez más cuestionamientos es el del impacto: ¿qué se entiende por impacto? ¿Quiénes y cómo se mide? ¿Cómo valorar un trabajo que, debido a distintas condiciones ajenas a la investigación misma, no impacta en la sociedad, en el momento de su publicación?

Este trabajo explora algunas tendencias temáticas, metodológicas y de producción de periodismo de investigación en el Cono sur (excluyendo Brasil) y las percepciones que informantes clave tienen sobre este en Argentina, Chile, Uruguay y Paraguay. Las principales limitaciones están dadas por el marco del cual surgen las investigaciones analizadas -aquellas finalistas o ganadoras del Premio a la Mejor Investigación Periodística en América Latina-. Otros trabajos, bajo distintos enfoques y recurriendo a distintas metodologías (estudios de casos o análisis comparado, por ejemplo) deberán ahondar en estas primeras aproximaciones al periodismo de investigación en estos países.

\section{Referencias bibliográficas}

Becerra, M. y Alfonso, A. (Comps.). (2007). La investigación periodística en la Argentina. Buenos Aires: Universidad Nacional de Quilmes.

Becerra, M. y Mastrini, G. (2006). Periodistas y magnates. Estructura y concentración de las industrias culturales en América Latina. Buenos Aires: Prometeo.

Becerra, M. y Mastrini, G. (2009). Los dueños de la palabra. Acceso, estructura y concentración de los medios en la América Latina del siglo XXI. Buenos Aires: Prometeo.

Bernstein, C. y Woodward, B. (1984). Todos los hombres del presidente. Bogotá: Oveja Negra.

Cañizález, A. (Coord.). (2006). Ojos frescos y bien abiertos. Apuntes sobre periodismo de investigación. Caracas: IPYS Venezuela.

Cárdena, F. y González, J. (2006). Los Watergates latinos: Prensa vs gobernantes corruptos. Bogotá: Ediciones B.

Hallin, D. y Papathanassopoulos, S. (2002). Political clientelism and the media: southern Europe and Latin America in comparative perspective. Media, culture \& society, 24(2), 175-195. 
Martínez, G. (Comp.). (2004). Periodismo de investigación. Fuentes, técnicas e informes. Buenos Aires: Ugerman.

Shoemaker, P. y Reese, S. (1996). Mediating the Message: Theories of Influences on Mass Media Content. Nueva York: Longman.

Waisbord, S. (2000). Watchdog Journalism in South America: News, Accountability and Democracy. Nueva York: Columbia University Press. 